\title{
Measuring the Group Velocity Dispersion of Higher Order Modes in Hollow Core Photonic Bandgap Fibre
}

\author{
T D Bradley, X Wang, E Numkam Fokoua, N V Wheeler, F Poletti, M N Petrovich \& D J Richardson \\ Optoelectronics Research Centre, University of Southampton, Southampton, SO17 1BJ, United \\ Kingdom tb1f13@soton.ac.uk
}

\begin{abstract}
We present for the first time the group velocity dispersion of multiple distinguishable modes propagating in 3, 7 and 19 cell hollow core photonic bandgap fibres. Measurements are made by direct phase extraction from spectral domain low coherence interferometry.
\end{abstract}

\section{Introduction}

Hollow core photonic bandgap fibres (HCPBGFs) have a range of properties including ultralow nonlinearity and latency, low propagation loss and the ability to guide multiple modes which makes them a candidate for a wide variety of applications. This potential includes the ability to enhance light matter interactions by filling the hollow core with a suitable medium, to ultrashort pulse delivery for manufacturing and medical applications, and more recently for telecommunications because of their ultralow nonlinearity and low latency ${ }^{1}$.

In order to fully exploit HC-PBGFs in these applications it is crucial to understand all the optical properties including attenuation, optical bandwidth, modal content and group velocity dispersion (GVD). To date great strides have been made in loss reduction, bandwidth optimisation, surface mode control and more recently volume upscaling to yield multi kilometre lengths ${ }^{2,3}$. As HC-PBGFs are generally few-moded, accurate characterisation of mode dependent properties is paramount and an area of great interest. In particular, the mode dependent GVD has never been experimentally investigated in detail for these fibres. Measurement of GVD in HC-PBGFs presents a challenge due to the relatively wide optical bandwidth of these fibres (up to $200 \mathrm{~nm}$ in latest generation fibres ${ }^{2}$ ) combined with the extremely large change in the GVD across the full bandwidth, and the potential for surface mode resonances to introduce very complex wavelength dependence. The transverse microstructure of a HC-PBGF has a strong impact on all the optical properties, the inherent difficulty in predicting the presence of features such as surface modes means that characterisation tools to measure the GVD are of paramount importance.

Here we present for the first time to our knowledge an experimental measurement of the GVD profiles of the modes propagating in 3, 7 and 19 cell HC-PBGFs. In this paper we firstly outline the experimental method and then go on to discuss the GVD profiles obtained for different optical modes propagating in 3,7 and 19 cell HC-PBGFs.

\section{Experimental method}

To determine GVD we use a low coherence Mach-Zehnder interferometer (Fig. 1).

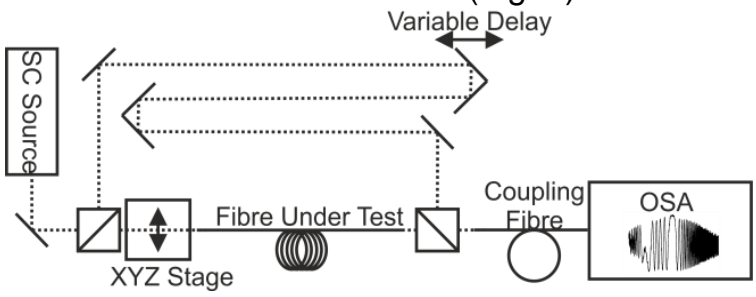

Fig. 1: Experimental set up of low coherence interferometer.

A supercontinuum source (Fianium WL-SC4002) spanning $400-2000 \mathrm{~nm}$ with $2 \mathrm{~W}$ average power is polarised (not shown in Fig. 1) and split into a reference arm (a variable free space delay line) and a measurement arm containing the HC-PBGF under test. The two beams are recombined and coupled into an optical spectrum analyser (OSA) via a large mode area fibre. From the measured spectral interference the phase is extracted and dispersion calculated $^{4}$. In order to identify the mode to which each interferogram belongs a $4 \%$ optical pick up is positioned (not shown in Fig. 1) after the recombining beam splitter cube and the sampled beam directed to a camera, allowing real time imaging of the propagating mode with simultaneous GVD measurement.

The spectral domain low coherence interferometry $(\mathrm{LCl})$ in this work directly extracts the phase from the full interferogram resulting in single shot broadband $(>100 \mathrm{~nm})$ and dense (10 points $/ \mathrm{nm})$ measurements. The phase extraction from the raw interferogram is a three stage process. This direct phase extraction has been previously validated on a number of different single mode fibres ${ }^{4}$, here we apply it to the case of few moded HC-PBGFs. Firstly the interferogram is normalised to the source spectrum as described by Eq. (1), examples of 
which can be seen in the black curves of the upper panel of Fig. 2.

$$
I_{N}=\frac{I_{\text {Int }}-\left(I_{F S}+I_{F i b r e}\right)}{2 \sqrt{\left(I_{F S} \cdot I_{F i b r e}\right)}}=V(\lambda) \cos (\phi[\lambda])
$$

The normalised inteferograms then have the envelope function removed and the cosine function inverted to obtain the phase curves (dashed blue) in the middle and lower panels of Fig. 2. The corresponding GVD (solid red curves) can then be straightforwardly obtained from the phase curves giving extremely dense wavelength sampling across the entire photonic bandgap (dashed black curve top panel). The spectral location of the centres of symmetry (CoS) corresponding to matched group delay between the two interferometer arms can be shifted by altering the free space path length. Adjusting the spectral location of the CoS (Top Panel Fig. 2) allows the spectral interference to be recorded over the whole bandgap and thus allowing the correspondingly large excursion in GVD to be measured. Each unique interferogram is processed and the phase extracted yielding separate GVD curves (solid red curves in middle and lower tier of Fig. 2). The multiple separate GVD curves are fitted with a cubic polynomial function to give a smooth continuous GVD profile.

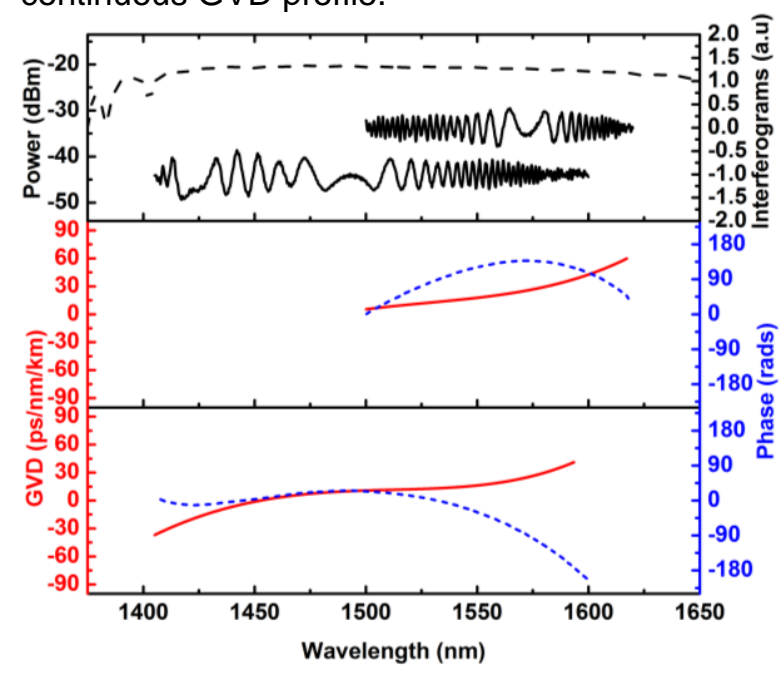

Fig. 2: Top: spectral power transmitted (dashed black) through $3 \mathrm{~m}$ long HC-PBGF sample and normalised interferograms (solid black) which have been offset for clarity, Middle \& Lower panels: show the extracted phase curve (dashed blue) and the GVD (solid red) from the corresponding interferogram.

LCI techniques with specialised algorithm processing $^{5}$ have been used to analyse the GVD of modes propagating in few mode fibres. The direct phase extraction method outlined here has particular advantages ideally suited for HC-PBGFs. HC-PBGFs typically have broad optical bandwidth combined with GVD which is highly divergent to both positive and negative values at the bandgap edges, and in general support multiple modes. LCl combined with the direct phase extraction used here allows for rapid single shot measurements which yield high resolution dispersion data due to the fact that the entire interferogram is utilised. This allows the large rapid variations in GVD in HC-PBGFs to be measured accurately. When this is combined with the ability to readily measure multiple interferograms spanning the entire bandgap and a wide range of GVD values the attractiveness of this technique for measuring GVD in HC-PBGFs becomes apparent.

\section{Results \& Discussion}

To demonstrate the applicability of direct phase extraction LCl to HC-PBGF, the GVD (solid red curve in Fig. 3 ) is measured in a 3 cell HC$\mathrm{PBGF}^{6}$ with a transmission window centred at $1550 \mathrm{~nm}$ (solid black curve in Fig. 3). The measured GVD demonstrates a typical $S$ shaped curve varying from $-500 \mathrm{ps} / \mathrm{nm} / \mathrm{km}$ to $500 \mathrm{ps} / \mathrm{nm} / \mathrm{km}$, this huge swing in GVD was measured using only 5 interferograms. At the short wavelength bandgap edge the presence of a surface mode group prevents further analysis (dashed line in top panel of Fig. 3).

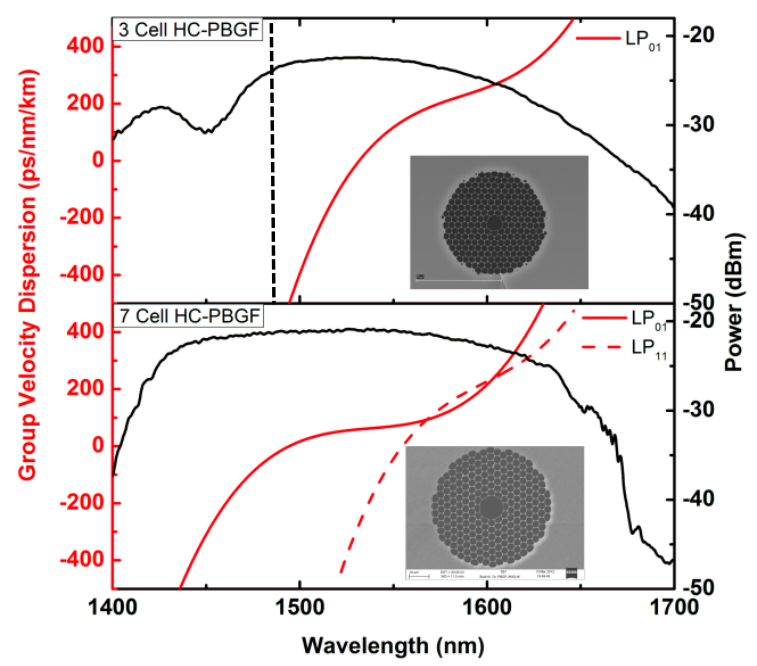

Fig. 3: Top: Group velocity dispersion (solid red) and optical transmission spectrum (solid black) of a 3 cell HC-PBGF. Lower: Group velocity dispersion of $\mathrm{LP}_{01}$ and $\mathrm{LP}_{11}$ mode (solid and dashed red) and optical transmission spectrum (solid black) of a 7 cell HC-PBGF.

A 7 cell HC-PBGF was investigated and the GVD curves associated with both the fundamental and $\mathrm{LP}_{11}$ mode were measured. The $L P_{01}$ mode GVD varies from $-500 \mathrm{ps} / \mathrm{nm} / \mathrm{km}$ to $500 \mathrm{ps} / \mathrm{nm} / \mathrm{km}$ across the full optical bandwidth. The $\mathrm{LP}_{11}$ GVD decreases below $-500 \mathrm{ps} / \mathrm{nm} / \mathrm{km}$ at $\sim 1510 \mathrm{~nm}$. Likely other modes are guided in this fibre over the length measured. However, these modes currently fall 
outside the maximum achievable measurement range set by the selected fibre length and experimental apparatus. Following the application to 3 and 7 cell HC-PBGFs we demonstrate the application of our technique to a 19 cell HC-PBGF ${ }^{1}$ (SEM in Fig 4) suitable for telecoms. The GVD has been measured for five modes (solid curves in Fig 4) including the $\mathrm{LP}_{01}$, two different $L P_{11}$ modes and two different $L P_{21}$ modes. The LCl mode dependent GVD show a typical S shape dispersion profile.

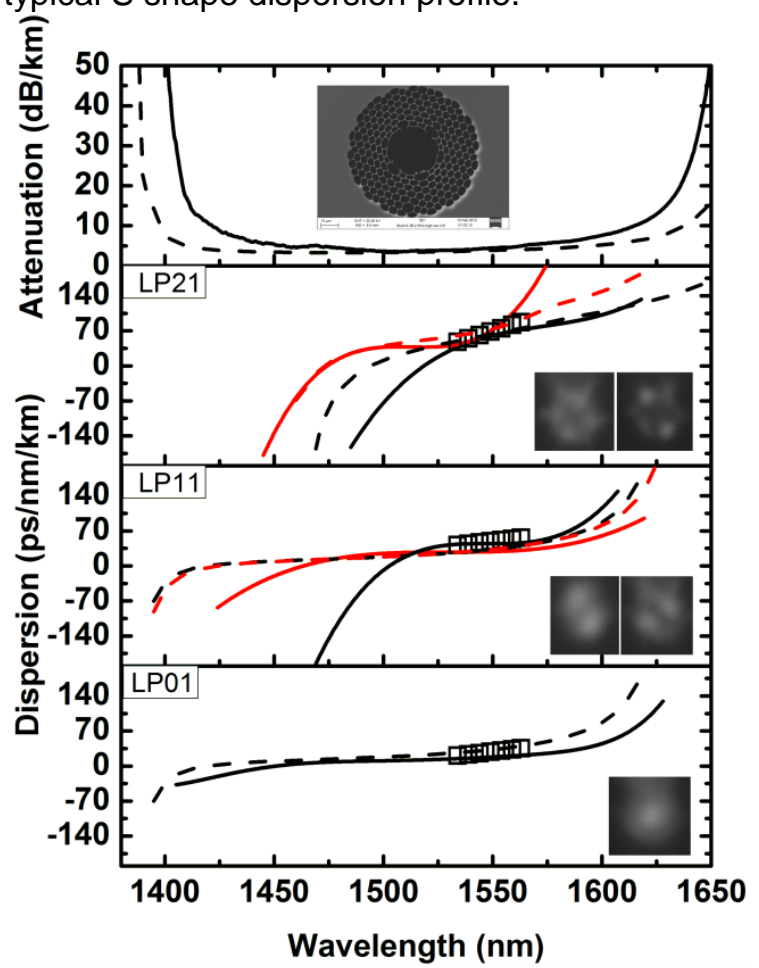

Fig. 4: Experimental (solid curves) and simulation data (dashed curves) for the HC-PBGF attenuation (top panel) and GVD of $L P_{21}$ (second panel), LP $P_{11}$ (third panel) and LP $P_{01}$ (bottom panel). Data points (open squares) show GVD data obtained through time of flight. Inset: SEM of HC-PBGF structure (top) and experimental mode profiles.

In the 19 cell HC-PBGF (Fig. 4) the $\mathrm{LP}_{01}$ has the lowest flattest GVD with a plateau region extending from $1470 \mathrm{~nm}$ to $1565 \mathrm{~nm}$ with a maximum GVD of $22 \mathrm{ps} / \mathrm{nm} / \mathrm{km}$ at $1565 \mathrm{~nm}$. For the higher order $L P_{11}$ and $L P_{21}$ modes the plateau region is spectrally narrower and has a higher GVD than the $\mathrm{LP}_{01}$ mode. The $\mathrm{LP}_{01}$ mode in the 3,7 and 19 cell HC-PBGF has substantial difference in the observed spectral extent of the plateau region and the gradient of GVD across the plateau. In the 3 cell HC-PBGF the $\mathrm{LP}_{01}$ plateau extends from $1565 \mathrm{~nm}$ to $1605 \mathrm{~nm}$ with a gradient of $2.5 \mathrm{ps} / \mathrm{nm}^{2} / \mathrm{km}$. The $L P_{01}$ mode in the 7 cell HC-PBGF has a plateau extending from $1505-1560 \mathrm{~nm}$ with a gradient of 0.8 $\mathrm{ps} / \mathrm{nm}^{2} / \mathrm{km}$. The 19 cell HC-PBGF LP ${ }_{01}$ GVD plateaus from $1470-1565 \mathrm{~nm}$ with a gradient of $0.14 \mathrm{ps} / \mathrm{nm}^{2} / \mathrm{km}$. There is an increase in the spectral extent of GVD plateau as the core defect is increased from 3 to 19 cell, increasing from $40 \mathrm{~nm}$ to $95 \mathrm{~nm}$. In addition there is a substantial reduction in the gradient of the GVD from $2.5 \mathrm{ps} / \mathrm{nm}^{2} / \mathrm{km}$ to $0.14 \mathrm{ps} / \mathrm{nm}^{2} / \mathrm{km}$ when the core defect is increased from 3 to 19 cell. This represents $a \sim 17 \times$ reduction in the gradient of the GVD combined with a more than two fold increase in the spectral extent of this gradient. For completeness, in Fig. 4 we include narrow band GVD obtained through time of flight (ToF) (averaged differential measurement) and simulations. Simulation data was obtained using a fully vectorial finite element solver (Comsol Multiphysics) which took as input a high-fidelity reproduction of the fibre cross-section from scanning electron micrographs. In the bandgap centre the ToF, simulations and LCl measurements all have good overlap. At the bandgap edges the $\mathrm{LCl}$ and simulated GVD measurements diverge because of the presence of surface mode resonances which are not identically portrayed in the simulated fibre attenuation.

\section{Conclusions}

We present for the first time to the best of our knowledge the GVD profiles of modes propagating in 3, 7 and 19 cell hollow core photonic bandgap fibres. A direct phase extraction technique previously developed by our group has been applied to the problem of determining the mode dependent GVD of HCPBGF for the first time. This technique will be of critical importance in the use of HC-PBGFs in pulse delivery and telecommunications applications.

\section{Acknowledgements}

This work was supported by the EU FP7ICT under grant 258033 (MODEGAP).

$X$. Wang is also with 'School of Electronic and Information Engineering, Beijing Jiaotong University, China.

\section{References}

[1] F. Poletti et al., "Towards high-capacity fibre-optic communications at the speed of light in vacuum," Nat. Photonics., Vol. 7, no. 4, p. 279 (2013).

[2] Y. Chen et al., "Demonstration of an $11 \mathrm{~km}$ Hollow Core Photonic Bandgap Fibre for Broadband Low-latency Data Transmission", Proc. OFC PDPTh5A.1., Los Angeles (2015)

[3] G. Jasion et al., "Volume Manufacturing of Hollow Core Photonic Bandgap Fibres: Challenges and Opportunities", Proc. OFC, W2A.37, Los Angeles (2015)

[4] G. M. Ponzo et al., "Fast and Broadband fiber dispersion measurements with dense wavelength sampling," Opts Exp., Vol. 14, no. 1, p. 943 (2014).

[5] R. Gabet et al., "Complete dispersion characterization of Few Mode Fibers by OCLI Technique", J. Lightwave Technol. Vol. 33, no 6, p. 1155 (2015).

[6] M. Petrovich et al., "Robustly single mode hollow core photonic bandgap fiber", Opts. Exp. Vol. 16, no. 6, p. 4337 (2008) 